\title{
Sensitivity of graphene humidity sensors
}

\author{
H. A. Zakaryan ${ }^{1}$, V.M. Aroutionian ${ }^{2}$ \\ ${ }^{1}$ Department of Physics of Semiconductors and Microelectronics, Yerevan State University \\ Yerevan, Armenia Republic of Armenia, Yerevan, 0025, 1 Alex Manoogian \\ zhayk91@gmail.com \\ ${ }^{2}$ Department of Physics of Semiconductors and Microelectronics, Yerevan State University \\ Yerevan, Armenia Republic of Armenia, Yerevan, 0025, 1 Alex Manoogian \\ aroutiounv1@yahoo.com
}

\begin{abstract}
Results of investigations of sensitivity of graphene to the humidity are reported. Comparison of properties of graphene and polymer humidity sensors are carried out. Graphene sensors has remarkable response at much lower water concentrations (started $0.01 \mathrm{~kg}$ water vapor in $1 \mathrm{~kg}$ air) compare to $\sim 40 \%$ of relative humidity for nanoporous polymer membranes. This resistivity is more than $150 \mathrm{GOhm}$ at $40 \%$ of the humidity and drops 7,5 times at $60 \%$ of the humidity. In our case the resistivity $\sim 100 \mathrm{KOhm}$ drops several times in the same range of the humidity. We think that it is connected with the graphene band opening phenomenon. During the adsorption water cause defects and open the band gap. The adsorbate also can transfer charge which depends on relative position of HOMO/LUMO of water and Dirac point of graphene.
\end{abstract}

Key words: graphene, sensors, humidity sensitivity, gap

\section{Introduction}

The humidity is everywhere in the environment, so it is important to precisely sense water molecules. Sensors, especially humidity sensors, are of great demand in industry and environmental fields, such as meteorological services, air conditioning, and electronics processing. Many efforts have been done to produce high performance humidity sensors [1, 2, 3]. Their working principles based on the change in electrical properties (conductivity or dielectric constant). Adsorbed water molecules can exchange electrons with a surface or change material's structure, causing a change in the resistivity of the material. New types of materials for manufacture of humidity sensors are carbon nanotubes and graphene.

Graphene is a single atom thick layer of sp2 hybridized carbon atoms. It was established that pristine graphene is a semimetal with zero band gap. This lack of an electron band gap is the major obstacle limiting the utilization of graphene in nano electronic devices. Some experiments claim that water molecules can open band gap of graphene after their adsorbtion on it.

\section{Analysis of existing data}

In this section we will describe the experimental data obtained by Fazel Yavari [5]. An environmental chamber with precise control of the absolute humidity level was used to control the amount of water adsorbed to the graphene surface. A bandgap of $\approx 0.206 \mathrm{eV}$ can be opened by simply exposing the sample to an absolute humidity level of $\approx 0.31 \mathrm{~kg} \mathrm{~kg}-1$ (i.e., $0.31 \mathrm{~kg}$ of water per $1 \mathrm{~kg}$ of dry air) in chamber. The effect is reversible and the band gap reduces to $\approx 0.029$ $\mathrm{eV}$ in vacuum. So, any process that induces a band gap would make it a relevant semiconducting material. Graphene samples were synthesized in [5] by chemical vapor deposition (CVD) on copper (Cu) foil.

In the beginning of hydration experiments, the resistivity of the graphene sample was measured in vacuum. Then It was exposed to four different levels $(0.022,0.065$, 0.152 and $0.312 \mathrm{~kg}$ of water per $\mathrm{kg}$ of air) of absolute humidity inside the chamber. For each value of humidity, the change in the film resistivity was measured as a function of time (Fig. 1). The adsorption of water on graphene surface is a relatively slow process and it takes several hours 
for the resistivity to saturate at each humidity level. In (Fig 2), the effect of the absolute humidity on the saturated value of the graphene film resistivity and the time taken to achieve $90 \%$ of the saturation resistivity are presented. The graphene resistivity shows a nonlinear dependence on the absolute humidity $(\mathrm{H})$ and increases as $\approx \mathrm{H} 0.25$. Conversely, the time to achieve $90 \%$ of the saturated resistivity decreases with increasing humidity as $\approx \mathrm{H}$ ($0.25)$. Thus, the rate of the adsorption of water molecules to the graphene surfaces affected by the absolute humidity level. Once the number density of water molecules on the graphene surfaces saturated, the resistivity value also saturate. Note that the absolute change in the resistivity can be as large as $\approx 150 \%$ for the case of $0.312 \mathrm{~kg}$ of water vapor per $1 \mathrm{~kg}$ of dry air.

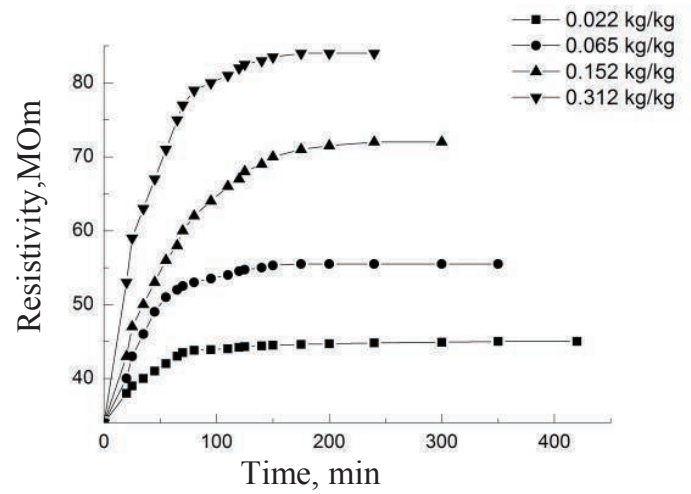

Fig.1. Rezistivity of graphene at different absolute humidity [5].

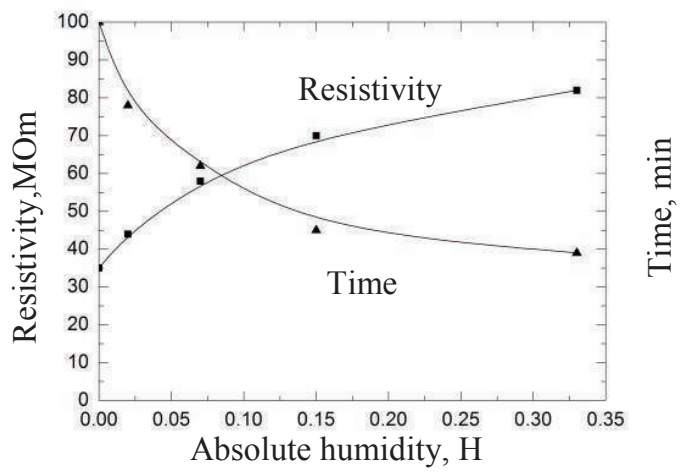

Fig.2.Dependance of saturated resistivity to absolute humidity and time [5].

To establish whether the sensitivity of the graphene sheet's resistivity to humidity is caused by the opening of a band gap in the material, we varied the temperature in the environmental chamber while keeping the absolute humidity constant. In the result, we can determine that an activation energy (a direct measure of band gap in intrinsic semiconductors) associated with electron transport in the graphene sheet exists.

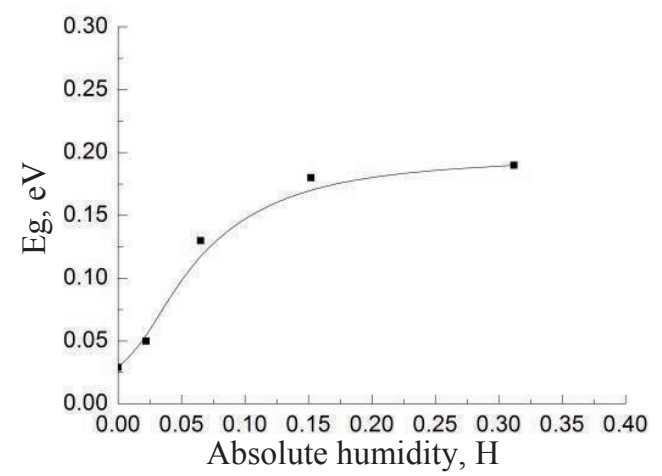

Fig.3. Dependance of band gap of graphene to absolute humidity [5].

For zero humidity in the chamber, the graphene nonzero band gap of $\approx 0.029 \mathrm{eV}$ is measured. This is probably a result of the impurity scattering, which leads to a small non zero band gap even in the absence of humidity or at substrate interactions. With an increase of the absolute humidity level measured the band gap Eg increases sharply following $a \approx H 0.33$ dependence and saturates at about $\mathrm{Eg} \approx 0.206$ $\mathrm{eV}$ at a humidity level of $\approx 0.312 \mathrm{~kg} \mathrm{~kg}-1$ (Fig. 3). Note that this effect is reversible. When the humidity level was reduced back to zero, the band gap dropped back to $\approx 0.029 \mathrm{eV}$. This was confirmed several times by repeated cycling of the humidity level in the $0-0.312 \mathrm{~kg} \mathrm{~kg}-1$ range. These experiments were also repeated for different graphene samples and the results were reproducible. 


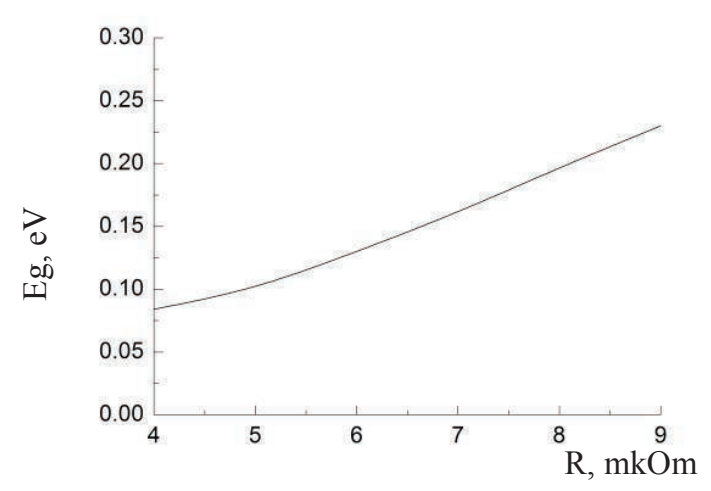

Fig.4.Dependance of band gap of graphene to resistivity

Using these measurements and the Wolfram Mathematica program we estimate the Eg dependence to resistivity, which is

$E_{g}=a+b R^{1.32}$,

where $a, b$ and $n$ are some constants. We see from (Fig. 4) that band gap increases when the $R$ resistance increases.

\section{Comparison of sensitivities}

During experiment the absolute humidity (or mixing ratio) was used, but in industry it is more convenient to use relative humidity. Therefore first we convert relative humidity to absolute humidity [13]. The mixing ratio (mass of water vapor/mass of dry gas) is calculated using :

$X=B \times P_{w} /\left(P_{\text {tot }}-P_{w}\right)[g / k g]$,

where $B=621.9907 \mathrm{~g} / \mathrm{kg}$

The value of $B$ depends on the gas. 621.9907 $\mathrm{g} / \mathrm{kg}$ is valid for air. In general the constant can be calculated using:

$\mathrm{B}=\mathrm{M}\left(\mathrm{H}_{2} \mathrm{O}\right) / \mathrm{M}($ gas $) \times 1000$

$\mathrm{PW}$ is water vapor pressure, which is calculated using

$$
\mathrm{RH}=\mathrm{P}_{\mathrm{w}} / \mathrm{P}_{\mathrm{ws}} \times 100 \%
$$

where Pws is saturation water vapor pressure.
There are many determination of sensitivity (response), but the most convenient, which we use is

$\mathrm{S}=\left(\mathrm{R}_{\mathrm{h}}-\mathrm{R}_{\mathrm{d}}\right) \times 100 / \mathrm{R}_{\mathrm{d}}$

Where RD is the resistivity of dry air, $\mathrm{RH}$ is the resistivity of humid air.

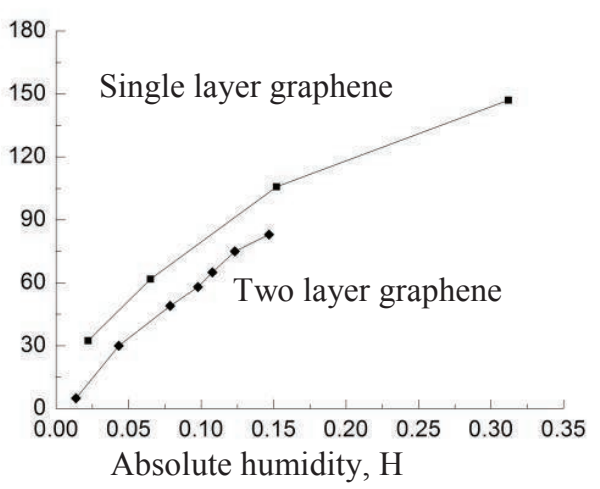

Fig.5.Sensitivity of single and two layer graphenes.

First we compare our results whit characteristic of two layer graphene [13]. The result is shown on Fig. 5. A single layer graphene is more sensitive than 2 layer graphene, and absolute change of resistivity is $150 \%$.We use this result to compare with other sensors sensitivities. For example, nanoporous polymer membranes have more than 150 GOhm resistivity at $40 \%$ of the humidity and drops 7,5 times at $60 \%$ of the humidity [6](Fig.6)

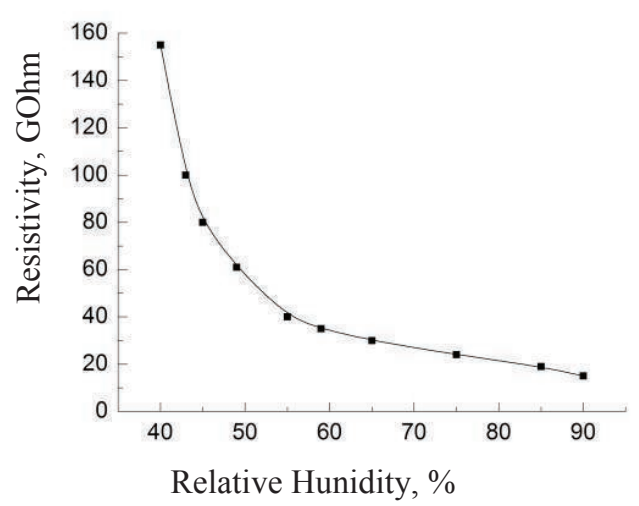

Fig.7. Resistivity of nanoporous polimer membrane to Relative humidity . 
The situation is practically the same for sensors made of hygroscopic polymer. Here the resistivity $\sim 10 \mathrm{MOhm}$ drops several times in the same range of the humidity [7].

\section{Band Gap opening}

Two effects can cause the band gap opening. The first is the breaking lattice symmetry. The molecular orbitals (HOMO/LUMO) mix with graphene orbitals creating the hybridization. This hybridization can cause asymmetric lattice, which dependent from site, where water molecule adsorbate on graphene cell. Using tight-bonding (TB) approach was calculated [8], that the energy dispersion of graphene with lattice constant $a$ is :

$\mathrm{E}^{ \pm}(\overrightarrow{\mathrm{k}})=\varepsilon_{2 \mathrm{p}} \mp \gamma \mathrm{f}(\overrightarrow{\mathrm{k}})$,

where

$$
\begin{aligned}
& \mathrm{f}(\overrightarrow{\mathrm{k}})=\sqrt{1+4 \cos \frac{\sqrt{3} \mathrm{k}_{\mathrm{x}} \mathrm{a}}{2} \cos \frac{\mathrm{k}_{\mathrm{y}} \mathrm{a}}{2}+4 \cos ^{2} \frac{\mathrm{k}_{\mathrm{x}} \mathrm{a}}{2}} \\
& \gamma=<\varphi_{\mathrm{A}}\left(\overrightarrow{\mathrm{r}}-\overrightarrow{\mathrm{R}_{\mathrm{A}}}\right)|\mathrm{H}| \varphi_{\mathrm{A}}\left(\overrightarrow{\mathrm{r}}-\overrightarrow{\mathrm{R}_{\mathrm{A}}}-\overrightarrow{\mathrm{R}_{1.1}}\right)> \\
& (\mathrm{i}=1,2,3)
\end{aligned}
$$

Here $\gamma$ is hopping integral, which show orbitals overlapping gauge, $\varepsilon_{2 \mathrm{p}}$ is the energy of the C- orbitals (a constant) [3]. From above, it is clear, that band gap will open if lattice symmetry breaks.

The second and probably more important effect is charge transfer $[9,10,11]$, which occurs due to the relative position in the DOS of the HOMO and LUMO of the adsorbate. If the HOMO is above the Fermi level of pure graphene (the Dirac point), there is a charge transfer to graphene. If the LUMO is below the Dirac point, charge will transfer to the molecule. In the case of water molecule, HOMO (mostly situated in oxygen atom) is below -4ev, LUMO (mostly situated in Hydrogen) is above $2 \mathrm{eV}$ (Fig7). Water can show both doping and acceptor features depending on what position and orientation molecule has. If molecule binds by HOMO orbitals, there is small charge transfer to graphene, due to all HOMO are occupied. But if molecule binds by LUMO, charge can transfer to adatom. In this case thermal energy also help electrons to transfer to adatom. So water mostly shows acceptor features, and this is the most possible orientation of the adsorbate.

\section{Conclusion}

It was proved that sensitivity increases due to band gap opening. There are two reasons of band gap opening, the one is that adsorbed molecules breaks symmetry of graphene lattice, another is charge transfer between molecule and graphene, in many cases water act as an acceptor. So, it was shown that graphene can be used to sense humidity with high precision, compare to 2 layer graphene and other types of sensors.

\section{References}

[1] F.Schedin, K.S.Novoselov, S.V.Morozov, P.Blake, D.Jiang, E.H.Hill, A.K.Geim. Detection of individual gas molecules adsorbed on graphene, condmat/0610809, (2006).

[2] Eds. R. Jakniso and O.K. Tan. Semiconducter gas sensors, WP Publishing et al.,(2013), 552pp (In particular, V.M Aroutiounian Porous silicon gas sensors, Ch. 12, pp 408-403).

[3] Encyclopedia of nanoseince and nanotechnology, CRS Press, (2012).

[4] V.M. Aroutiounian. Armenian J. Phys.,6,141(2013).

[5] F.Yaiari. Tunable bandgap by graphene by the control adsorption of water molecules. Small pp. 2535-2538, (2010).

[6] B.Yang. Sensors and Actuators B, 114,pp.254 (2006).

[7] Ohmic instrument co. , http://www.ohmicinstruments.com/pdf/UPS500.pdf.

[8] G.Gui, J.Li, J.Zhong. Phys.Rev. B, 78, 075435 (2008).

[9] K. Nakada, A. Ishii. DFT Calculation for Adatom Adsorption on Graphene, Graphene Simulation, Prof. Jian Gong (Ed.), ISBN: 978953-307-556-3, InTech, (2011). 
[10] H.S Wahablnternational Journal of Basic \& Applied Sciences, 12,234, (2012).

[11] O. Leenaerts. arXiv:0710.1757v1 (2007).

[12] W. Wagner, A. Pru. The IAPWS Formulation for the Thermodynamic Properties of Ordinary Water Substance for General and Scientific Use, J. Phys. Chem. Reference Data,31, (2002)

[13] A. Ghosh, D. J. Late. arXiv:0905.2852 (2009). 